\title{
Reliability of Wireless Sensor Network with Sleeping Nodes ${ }^{\star}$
}

\author{
Vladimir V. Shakhov ${ }^{1}$ and Hyunseung $\mathrm{Choo}^{2, \star \star}$ \\ ${ }^{1}$ Institute of Computational Mathematics and Mathematical Geophysics of SB RAS \\ Novosibirsk 630090, Russia \\ shakhov@skku.edu \\ ${ }^{2}$ School of Information and Communication Engineering, Sungkyunkwan University \\ Suwon 440-746, South Korea \\ choo@ece.skku.ac.kr
}

\begin{abstract}
Energy efficiency is an important technology of sensor networks development. In this paper we offer a method of sensors lifetime maximizing under required level of network reliability. The optimizing statement is offered. As example, we consider homogeneous sensor networks with topology like tree. Other example concerns ZigBee technology. It shown that heterogeneous network can be improved by power elements reassignments. If a sensor network topology is fixed then the most critical nodes can be detected and improved.
\end{abstract}

Keywords: Wireless Sensor Networks, Reliability, and Sleeping Time.

\section{Introduction}

A cost of sensor components is a critical consideration in the design of practical sensor networks. A cost of sensor network increases with sensor battery power. It is often economically advantageous to discard a sensor rather then sensor recharging. By this reason a battery power is usually a scare component in wireless devices. On the other hand, sensor lifetime depends on battery lifetime. Thus, energy efficiency is an important direction of sensor networks investigations [1].

A widely employed energy-saving approach consists in placing sensors in sleeping mode. During sleeping time a sensor can be used for data transmission or other services like environment monitoring. Hence, network reliability degrades. But sensor power consumption is very low under the mentioned mode. Hence, sensors lifetime increases. Thus, it is necessary to find a trade-off between sleeping time and network reliability. Many researchers are currently engaged in

\footnotetext{
* This research was supported by the MIC (Ministry of Information and Communication), Korea, under the ITRC (Information Technology Research Center) support program supervised by the IITA (Institute of Information Technology Assessment),IITA-2006-(C1090-0603-0046).

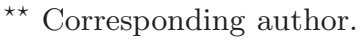


energy-saving mechanisms developing that are based on sleeping mode application. Investigations are focused on scheduling schemes for sensors lifetime maximization [2] or protocol for network throughput increasing [3. An interrelation of sensor battery lifetime and network reliability has not been considered. To fill this gap we offer a method of sensors lifetime maximizing under required level of network reliability.

The rest of paper is organized as follows. In section 2 we discuss a concept of reliability and state an optimizing problem for sensor lifetime maximization. In 3.1 , we propose the problem solution for homogenous sensor networks. In 3.2 we produce some details for ZigBee technology (IEEE 802.15.4.) and consider the problem for heterogeneous. Section 4 is the final conclusion.

\section{Sensor Reliability and Network Reliability}

As it was said above a sensor network is a set of low-cost and low-power wireless sensor. Let us designate the sensor lifetime as $T$ and the total sensor sleeping time as $S$. Thus, the duration of sensor active state is $T-S$. Assume that a sensor is randomly switched from active state to sleep state and inversely. From here, the probability $p$ of single sensor availability can be calculated as

$$
p=\frac{T-S}{T}=1-\frac{S}{T} .
$$

If a sensor network is modeled by a graph then the probability $p$ is a reliability of graph vertex. Thus, sensor networks with sleeping nodes are modeled by a graph with unreliable vertices. Actually, a wireless channel is also unreliable due to interference, fading, shading and so on. But a distance between sensors is not large. So, not reduce generality, we assume that edges of graph above are absolutely reliable. The probability of graph (network) connectivity is known as network reliability, $R$. Let $n$ is the total number of sensors in a network. It can be shown in the trivial way that network reliability is a polynomial of degree $n$ in the variable $p$. Let us remark we consider the connection for active nodes. Thus, the mentioned reliability reflects the connectivity probability of non-sleeping nodes.

The wished sensor lifetime $T$ is usually given. It is defined by applications. But if sleeping time is maximized then the capacity of sensor battery is reduced. It also reduces a cost of sensor networks. But from (1) we can see that large sleeping time is a reason of network unreliability. Thus, it is necessary to minimize sensor availability saving given level of network reliability, $C$. Here $C \in[0,1]$. Continuing this line of reasoning, we get the following optimization problem

$$
\begin{aligned}
& p \rightarrow \min \\
& R(p) \geq C
\end{aligned}
$$

Let us remark the problem is formulated for homogeneous network nodes. The parameter $p$ is same for all sensors. 


\section{Reliability Calculations}

\subsection{Reliability of Homogeneous Sensor Network}

In the optimization statement above the criterion is a monotone continuous growing function in p. It is obvious that a reliability polynomial monotonically grows when $\mathrm{p}$ runs over the set $[0 \ldots 1]$. Thus, the solution of considered optimization problem is reached on area boundary defined by the function $R(p)$. Therefore, the optimal $p$ is a root of equation

$$
R(p)=C .
$$

Let us consider the following example. Two terminals are connected by $n$ sequential sensors. Thus, the sensor network topology is a tree then the function of reliability (3) equals $R(p)=p^{n}$, here we do not take into account failures of terminals.

If we solve the equation $p^{n}=C$ then we find an optimal solution of the problem (2). Thus, the optimal parameter $p$ is

$$
p_{o p t}=C^{\frac{1}{n}} .
$$

From here, the optimal sleeping time equals

$$
S=T\left(1-C^{\frac{1}{n}}\right) .
$$

In case of huge number of sensors and arbitrary network topology the exact solution can be impractical. Numerical methods can be used.

\subsection{Reliability of Heterogeneous Sensor Network}

The approach above with insignificant modification can be used for heterogeneous nodes. Now the function of reliability in (2) is a function on a few variables. In some practical cases the optimal topology has to be heterogeneous. Actually, if a sensor network topology is fixed then the most critical nodes can be detected and improved. We demonstrate it on the following example. Let us consider a Personal Area Network (PAN) technology known as ZigBee. A ZigBee network consists of three types of logical units 4. There are ZigBee Coordinator (ZC), ZigBee Router (ZR) and ZigBee End Device (ZED). ZC is PAN coordinator. The coordinator functions depend on network applications. ZED is data terminal equipment. ZR provides a connection of ZEDs and ZC.

Assume ZC and ZED are connected by three ZigBee Routers A,B, and C. Not reduce generality we consider the case of absolutely reliable $\mathrm{ZC}$ and ZEDs and unreliable ZR. Let a network topology be as shown on the adjacency matrix.

\section{ZC A B C ZED}

$\begin{array}{cccccc}\mathbf{Z C} & 0 & 1 & 0 & 0 & 0 \\ \mathbf{A} & 1 & 0 & 1 & 1 & 0 \\ \mathbf{B} & 0 & 1 & 0 & 0 & 1 \\ \mathbf{C} & 0 & 1 & 0 & 0 & 1 \\ \mathbf{Z E D} & 0 & 0 & 1 & 1 & 0\end{array}$


Let $p_{A}$ be the probability of active status of ZigBee Router $\mathrm{A}, p_{B}$ be the probability of active status of $\mathrm{ZR} \mathrm{B}$, and $p_{C}$ be the probability of active status of ZR C.

The network reliability is

$$
R\left(p_{A}, p_{B}, p_{C}\right)=p_{A}\left(1-\left(1-p_{B}\right)\left(1-p_{C}\right)\right)
$$

Hence the equations (2), (3) look as follows

$$
\begin{gathered}
p_{A}+p_{B}+p_{C} \rightarrow \min \\
p_{A}\left(p_{B}+p_{C}-p_{B} p_{C}\right) \geq C
\end{gathered}
$$

The expressions above can be simplified. From the reasons of symmetry we get $p_{B}=p_{C}$. Now the reader will have no difficulty in finding the optimal solution. It is easy to see that the optimal $p_{A}$ exceeds the probabilities $p_{B}$ and $p_{C}$. Thus, for considered network topology the optimal sleeping times of network nodes are not the same. Hence, the optimal network structure are not homogeneous.

\section{Conclusion}

In this paper, we investigated one of the methods of cost reduction for sensors network. The offered technique allows to minimize battery capacity and calculate the optimal sleeping time of sensors. At the same time the required level of reliability is kept. The problem of sensor network reliability is reduced to an optimizing problem with monotone and continuous functions. Solutions of the problem can be calculated by Lagrange approach. In some cases a heterogeneous assignment of power is preferable. Actually, if a sensor network topology is fixed then the most critical nodes can be detected. And a network topology can be improved by power elements reassignments. In the future it is reasonable to investigate the problem of reliability maximization under given cost of sensors.

\section{References}

1. Akyildiz, I., Su, W., Sankarasubramaniam, Y., Cayirci, E.: A survey on sensor networks. Communications Magazine, Vol. 40, Issue 8. IEEE (2002) 102 - 114

2. Subramanian, R., Fekri, F.: Sleep scheduling and lifetime maximization in sensor networks: fundamental limits and optimal solutions. Proceeding of IPSN. IEEE (2006) $218-225$.

3. Chiasserini, C.-F., Garetto, M.: An Analytical Model for Wireless Sensor Networks with Sleeping Nodes. Transactions on Mobile Computing, Vol. 5, Issue 12. IEEE (2006) $706-1718$.

4. Vargauzin, V.: Wireless sensor networks based on IEEE 802.15.4, TeleMultiMedia, Vol. 6. Telesputnik (2005) (in Russian). 